\title{
Existence and uniqueness of mild solutions for nonlinear hybrid Caputo fractional integro-differential equations via fixed point theorems
}

\author{
Abderrahim Guerfia ${ }^{a}$ Abdelouaheb Ardjounib \\ ${ }^{a}$ Department of Mathematics, Annaba University, Annaba, Algeria. \\ ${ }^{b}$ Department of Mathematics and Informatics, Souk Ahras University, Souk Ahras, Algeria.
}

\begin{abstract}
We prove the existence and uniqueness of mild solutions for initial value problems of nonlinear hybrid first order Caputo fractional integro-differential equations. The main tool employed here is the Krasnoselskii and Banach fixed point theorems. An example is also given to illustrate the main results. In addition, the case of the Higher order Caputo fractional integro-differential equations is studied.
\end{abstract}

Keywords: Hybrid fractional integro-differential equations, Fixed point theorems, Existence, Uniqueness. 2010 MSC: 26A33, 34A12, 45G05, 47H10.

\section{Introduction}

Fractional differential equations arise from a variety of applications including in various fields of science and engineering. In particular, problems concerning qualitative analysis of fractional differential equations have received the attention of many authors, see [1]-[14], [16]-[20] and the references therein.

Hybrid differential equations involve the fractional derivative of an unknown function hybrid with the nonlinearity depending on it. This class of equations arises from a variety of different areas of applied mathematics and physics, e.g., in the deflection of a curved beam having a constant or varying cross section, a three-layer beam, electromagnetic waves or gravity driven flows and so on [6], [7], [9]-[11], [16], [17].

Email addresses: abderrahimg21@gmail.com (Abderrahim Guerfi), abd_ardjouni@yahoo.fr (Abdelouaheb Ardjouni) 
Recently, Dhage [7] discussed the following first order hybrid differential equation with mixed perturbations of the second type

$$
\left\{\begin{array}{l}
\frac{d}{d t}\left[\frac{u(t)-k(t, u(t))}{f(t, u(t))}\right]=g(t, u(t)), t \in\left[t_{0}, t_{0}+a\right] \\
u\left(t_{0}\right)=x_{0} \in \mathbb{R}
\end{array}\right.
$$

where $\left[t_{0}, t_{0}+a\right]$ is a bounded interval in $\mathbb{R}$ for some $t_{0}, a \in \mathbb{R}$ with $a>0, f:\left[t_{0}, t_{0}+a\right] \times \mathbb{R} \rightarrow \mathbb{R} \backslash\{0\}$ and $k, g:\left[t_{0}, t_{0}+a\right] \times \mathbb{R} \rightarrow \mathbb{R}$ are continuous functions. He developed the theory of hybrid differential equations with mixed perturbations of the second type and provided some original and interesting results.

Zhao et al. [20] discussed the following boundary value problem of nonlinear fractional differential equations with mixed perturbations of the second type

$$
\left\{\begin{array}{l}
{ }^{C} D_{0^{+}}^{\alpha}\left[\frac{u(t)-k(t, u(t))}{f(t, u(t))}\right]=g(t, u(t)), t \in J=[0, T], \\
a\left[\frac{u(t)-k(t, u(t))}{f(t, u(t))}\right]_{t=0}+b\left[\frac{u(t)-k(t, u(t))}{f(t, u(t))}\right]_{t=T}=c
\end{array}\right.
$$

where $0<\alpha \leq 1,{ }^{C} D_{0^{+}}^{\alpha}$ is the Caputo fractional derivative, $f: J \times \mathbb{R} \rightarrow \mathbb{R} \backslash\{0\}$ and $k, g: J \times \mathbb{R} \rightarrow \mathbb{R}$ are continuous functions, $a, b$ and $c$ are real constants with $a+b \neq 0$. They established an existence theorem for the boundary value problem under mixed Lipschitz and Carathéodory conditions by using the fixed point theorem in Banach algebra due to Dhage.

In [1], Ardjouni and Djoudi studied the existence and approximation of solutions for the following initial value problem of nonlinear hybrid Caputo fractional integro-differential equations

$$
\left\{\begin{array}{l}
{ }^{C} D_{0^{+}}^{\alpha}\left(\frac{u(t)}{p(t)+\frac{1}{\Gamma(\beta)} \int_{0}^{t}(t-s)^{\beta-1} g(s, u(s)) d s}\right)=f(t, u(t)), t \in J=[0, a], \\
u(0)=p(0) \theta
\end{array}\right.
$$

where $0<\alpha \leq 1,0<\beta \leq 1, \theta \in \mathbb{R}, g, f: J \times \mathbb{R} \rightarrow \mathbb{R}$ are continuous functions and $p: J \rightarrow \mathbb{R}$ is a continuous function. By using the Dhage iteration principle, the authors obtained the existence and approximation of solutions under weaker partially continuity and partially compactness type conditions.

In this paper, we discuss the existence and uniqueness of mild solutions for the following initial value problem of nonlinear hybrid first order Caputo fractional integro-differential equations

$$
\left\{\begin{array}{l}
{ }^{C} D_{0^{+}}^{\alpha}\left(\frac{u(t)-f(t, u(t))}{p(t)+\frac{1}{\Gamma(\beta)} \int_{0}^{t}(t-s)^{\beta-1} g(s, u(s)) d s}\right)=h(t, u(t)), t \in[0, T], \\
u(0)=f(0, u(0))+p(0) \theta,
\end{array}\right.
$$

where ${ }^{C} D_{0^{+}}^{\alpha}$ denotes the Caputo fractional derivative of order $\alpha \in(0,1), \beta \in(0,1), \theta \in \mathbb{R}, p:[0, T] \rightarrow \mathbb{R}$ and $f, g, h:[0, T] \times \mathbb{R} \rightarrow \mathbb{R}$ are continuous functions with $p(t)+I_{0^{+}}^{\beta} g(t, u(t)) \neq 0$. To show the existence and uniqueness of mild solutions, we transform (1) into an integral equation and then use the Krasnoselskii and Banach fixed point theorems. Also, we provide an example to illustrate our obtained results. Finally, we study the Higher order Caputo fractional integro-differential equations.

\section{Preliminaries}

Let $C([0, T], \mathbb{R})$ be the Banach space of all real-valued continuous functions defined on the compact interval $[0, T]$, endowed with the norm

$$
\|u\|=\sup _{t \in[0, T]}|u(t)| .
$$

$L^{1}([0, T], \mathbb{R})$ denotes the space of Lebesgue integrable functions on $[0, T]$ equipped with the norm $\|\cdot\|_{L^{1}}$ defined by

$$
\|u\|_{L^{1}}=\int_{0}^{T}|u(s)| d s .
$$


We consider the following set of assumptions.

$\left(\mathrm{A}_{1}\right)$ There exists a constant $K_{f}>0$ such that

$$
|f(t, u)-f(t, v)| \leq K_{f}|u-v|
$$

for all $t \in[0, T]$ and $u, v \in \mathbb{R}$.

$\left(\mathrm{A}_{2}\right)$ There exist functions $H, G \in L^{1}\left([0, T], \mathbb{R}_{+}\right)$such that

$$
|h(t, u)| \leq H(t) \text { and }|g(t, u)| \leq G(t), t \in[0, T] .
$$

$\left(\mathrm{A}_{3}\right)$ There exists a constant $K_{p}>0$ such that

$$
\left|p\left(t_{2}\right)-p\left(t_{1}\right)\right| \leq K_{p}\left|t_{2}-t_{1}\right| \text { for all } t_{1}, t_{2} \in[0, T] .
$$

$\left(\mathrm{A}_{4}\right)$ There exist constants $K_{h}, K_{g}>0$ such that

$$
|h(t, u)-h(t, v)| \leq K_{h}|u-v| \text { and }|g(t, u)-g(t, v)| \leq K_{g}|u-v|
$$

for all $t \in[0, T]$ and $u, v \in \mathbb{R}$.

We introduce some basic definitions and necessary lemmas related to fractional calculus and fixed point theorems that will be used throughout this paper.

Definition 2.1 ([13]). The left sided Riemann-Liouville fractional integral of order $\alpha>0$ of a function $u:[0, T] \rightarrow \mathbb{R}$ is given by

$$
I_{0^{+}}^{\alpha} u(t)=\frac{1}{\Gamma(\alpha)} \int_{0}^{t}(t-s)^{\alpha-1} u(s) d s,
$$

where $\Gamma$ denotes the gamma function.

Definition 2.2 ([13]). Let $n-1<\alpha<n$. The left sided Riemann-Liouville fractional derivative of order $\alpha$ of a function $u:[0, T] \rightarrow \mathbb{R}$ is defined by

$$
D_{0^{+}}^{\alpha} u(t)=\frac{d^{n}}{d t^{n}} I_{0^{+}}^{n-\alpha} u(t)=\frac{1}{\Gamma(n-\alpha)} \frac{d^{n}}{d t^{n}} \int_{0}^{t}(t-s)^{n-\alpha-1} u(s) d s, t>0,
$$

provided the right side integral is pointwise defined on $[0, T]$. In particular, if $0<\alpha<1$, then

$$
D_{0^{+}}^{\alpha} u(t)=\frac{d}{d t} I_{0^{+}}^{1-\alpha} u(t)=\frac{1}{\Gamma(1-\alpha)} \frac{d}{d t} \int_{0}^{t} \frac{u(s)}{(t-s)^{\alpha}} d s, t>0 .
$$

Definition $2.3([13])$. Let $n-1<\alpha<n$. The left sided Caputo fractional derivative of order $\alpha>0$ of a function $u \in C^{n}([0, T], \mathbb{R})$ is given by

$$
{ }^{C} D_{0^{+}}^{\alpha} x(t)=I_{0^{+}}^{n-\alpha} x^{(n)}(t)=\frac{1}{\Gamma(n-\alpha)} \int_{0}^{t}(t-s)^{n-\alpha-1} x^{(n)}(s) d s, t>0 .
$$

In particular, if $0<\alpha<1$, then

$$
{ }^{C} D_{0^{+}}^{\alpha} u(t)=I_{0^{+}}^{1-\alpha} u^{\prime}(t)=\frac{1}{\Gamma(1-\alpha)} \int_{0}^{t} \frac{u^{\prime}(s)}{(t-s)^{\alpha}} d s, t>0 .
$$

Moreover, the Caputo derivative of a constant is equal to zero.

Lemma $2.4([13])$. Let $\alpha>0$ and $u \in C^{n}([0, T], \mathbb{R})$. Then

1) ${ }^{C} D_{0^{+}}^{\alpha} I_{0^{+}}^{\alpha} u(t)=u(t)$.

2) $I_{0^{+}}^{\alpha C} D_{0^{+}}^{\alpha} u(t)=u(t)-\sum_{k=0}^{n-1} \frac{u^{(k)}(0)}{k !} t^{k}$.

In particular, when $\alpha \in(0,1), I_{0^{+}}^{\alpha C} D_{0^{+}}^{\alpha} u(t)=u(t)-u(0)$. 
From the definition of the Caputo derivative, we can obtain the following lemma.

Lemma $2.5([13])$. Let $n-1<\alpha<n$ and $u \in C^{n}([0, T], \mathbb{R})$. Then

$$
I_{0^{+}}^{\alpha C} D_{0^{+}}^{\alpha} u(t)=u(t)+c_{0}+c_{1} t+c_{2} t^{2}+\ldots+c_{n-1} t^{n-1},
$$

for some $c_{k} \in \mathbb{R}, k=0,1,2, \ldots, n-1$.

In particular, when $\alpha \in(0,1), I_{0^{+}}^{\alpha} D_{0^{+}}^{\alpha} u(t)=u(t)+c_{0}$.

The following Krasnoselskii's fixed point theorem is useful in the proof of our main results.

Theorem 2.6 (Krasnoselskii's fixed point theorem [15]). Let $\mathbb{M}$ be a non-empty closed bounded convex subset of a Banach space $(\mathbb{B},\|\|$.$) . Suppose that \mathcal{A}$ and $\mathcal{B}$ map $\mathbb{M}$ into $\mathbb{B}$ such that

(i) $\mathcal{A} x+\mathcal{B} y \in \mathbb{M}$ for all $x, y \in \mathbb{M}$,

(ii) $\mathcal{A}$ is continuous and compact,

(iii) $\mathcal{B}$ is a contraction with constant $r<1$.

Then there is $z \in \mathbb{M}$, with $\mathcal{A} z+\mathcal{B} z=z$.

\section{Main results}

In this section, we discuss the existence and uniqueness results for the initial value problems (1).

Let us start by defining what we mean by a mild solution of the problem (1).

Definition 3.1. A function $u \in C([0, T], \mathbb{R})$ is said to be a mild solution of the problem (1) if $u$ satisfies the corresponding integral equation of (1).

For the existence and uniqueness of solutions for the problem (1), we need the following lemma.

Lemma 3.2. $u \in C([0, T], \mathbb{R})$ is a mild solution of (1) if $u$ satisfies

$$
\begin{aligned}
u(t) & =\left(p(t)+\frac{1}{\Gamma(\beta)} \int_{0}^{t}(t-s)^{\beta-1} g(s, u(s)) d s\right) \\
& \times\left(\frac{1}{\Gamma(\alpha)} \int_{0}^{t}(t-s)^{\alpha-1} h(s, u(s)) d s+\theta\right)+f(t, u(t)) .
\end{aligned}
$$

Proof. Let $u$ be a solution of the problem (1). Applying the Riemann-Liouville fractional integral $I_{0^{+}}^{\alpha}$ on both sides of (1), by Lemma 2.5 , then we obtain

$$
\frac{u(t)-f(t, u(t))}{p(t)+\frac{1}{\Gamma(\beta)} \int_{0}^{t}(t-s)^{\beta-1} g(s, u(s)) d s}=I_{0^{+}}^{\alpha} h(t, u(t))+c,
$$

for some $c \in \mathbb{R}$. So, we get

$$
\begin{aligned}
u(t) & =\left(p(t)+\frac{1}{\Gamma(\beta)} \int_{0}^{t}(t-s)^{\beta-1} g(s, u(s)) d s\right) \\
& \times\left(\frac{1}{\Gamma(\alpha)} \int_{0}^{t}(t-s)^{\alpha-1} h(s, u(s)) d s+c\right)+f(t, u(t)) .
\end{aligned}
$$

Substituting $t=0$ in the above equality, we have

$$
u(0)=p(0) c+f(0, u(0)) .
$$

The condition $u(0)=f(0, u(0))+p(0) \theta$ implies that

$$
c=\theta .
$$

Substituting (4) in (3) we get the integral equation (2). 
Now we will give the following existence and uniqueness theorems for the initial value problem (1).

Theorem 3.3. Assume that hypotheses $\left(A_{1}\right)-\left(A_{3}\right)$ hold. Furthermore, if

$$
K_{f}<1,
$$

then the initial value problem (1) has a mild solution defined on $[0, T]$.

Proof. Set $\mathbb{B}=C([0, T], \mathbb{R})$ and define a subset $\mathbb{M}$ of $\mathbb{B}$ by

$$
\mathbb{M}=\{u \in \mathbb{B},\|u\| \leq N\},
$$

where

$$
N=K_{f} N+F_{0}+\left(K_{p} T+|p(0)|+\frac{T^{\beta}\|G\|_{L^{1}}}{\Gamma(\beta+1)}\right)\left(\frac{T^{\alpha}\|H\|_{L^{1}}}{\Gamma(\alpha+1)}+|\theta|\right),
$$

with $F_{0}=\sup _{t \in[0, T]}|f(t, 0)|$. Clearly, $\mathbb{M}$ is a closed, convex and bounded subset of the Banach space $\mathbb{B}$.

Define two operators $\mathcal{A}, \mathcal{B}: \mathbb{M} \rightarrow \mathbb{B}$ by

$$
\begin{aligned}
(\mathcal{A} u)(t) & =\left(p(t)+\frac{1}{\Gamma(\beta)} \int_{0}^{t}(t-s)^{\beta-1} g(s, u(s)) d s\right) \\
& \times\left(\frac{1}{\Gamma(\alpha)} \int_{0}^{t}(t-s)^{\alpha-1} h(s, u(s)) d s+\theta\right), t \in[0, T],
\end{aligned}
$$

and

$$
(\mathcal{B} u)(t)=f(t, u(t)), t \in[0, T] .
$$

Now, (2) is equivalent to the operator equation

$$
u(t)=(\mathcal{A} u)(t)+(\mathcal{B} u)(t), t \in[0, T] .
$$

We shall use Krasnoselskii's fixed point theorem to prove there exists at least one fixed point of the operator $\mathcal{A}+\mathcal{B}$ in $\mathbb{M}$. The proof will be given in several steps.

Step 1. We prove that $\mathcal{B}$ is a contraction with constant $K_{f}<1$. Let $u, v \in \mathbb{M}$. Then by $\left(\mathrm{A}_{1}\right)$, we get

$$
\begin{aligned}
|(\mathcal{B} u)(t)-(\mathcal{B} v)(t)| & =|f(t, u(t))-f(t, v(t))| \leq K_{f}|u(t)-v(t)| \\
& \leq K_{f}\|u-v\|
\end{aligned}
$$

for all $t \in[0, T]$. Taking supremum over $t$, then we have

$$
\|\mathcal{B} u-\mathcal{B} v\| \leq K_{f}\|u-v\|
$$

for all $u, v \in \mathbb{M}$. Thus, by (5), $\mathcal{B}$ is a contraction operator on $\mathbb{M}$ with constant $K_{f}<1$.

Step 2. We prove $\mathcal{A}$ is a compact operator on $\mathbb{M}$ into $\mathbb{B}$. It is enough to prove that $\mathcal{A}(\mathbb{M})$ is a uniformly bounded and equicontinuous set in $\mathbb{B}$. On the one hand, let $u \in \mathbb{M}$ be arbitrary. Then by $\left(\mathrm{A}_{2}\right)$, we get

$$
\begin{aligned}
|(\mathcal{A} u)(t)| & \leq\left(|p(t)|+\frac{1}{\Gamma(\beta)} \int_{0}^{t}(t-s)^{\beta-1}|g(s, u(s))| d s\right) \\
& \times\left(\frac{1}{\Gamma(\alpha)} \int_{0}^{t}(t-s)^{\alpha-1}|h(s, u(s))| d s+|\theta|\right) \\
& \leq\left(K_{p} t+|p(0)|+\frac{1}{\Gamma(\beta)} \int_{0}^{t}(t-s)^{\beta-1}|G(s)| d s\right) \\
& \times\left(\frac{1}{\Gamma(\alpha)} \int_{0}^{t}(t-s)^{\alpha-1}|H(s)| d s+|\theta|\right) \\
& \leq\left(K_{p} T+|p(0)|+\frac{T^{\beta}\|G\|_{L^{1}}}{\Gamma(\beta+1)}\right)\left(\frac{T^{\alpha}\|H\|_{L^{1}}}{\Gamma(\alpha+1)}+|\theta|\right)
\end{aligned}
$$


for all $t \in[0, T]$. Taking supremum over $t$, we obtain

$$
\|\mathcal{A} u\| \leq\left(K_{p} T+|p(0)|+\frac{T^{\beta}\|G\|_{L^{1}}}{\Gamma(\beta+1)}\right)\left(\frac{T^{\alpha}\|H\|_{L^{1}}}{\Gamma(\alpha+1)}+|\theta|\right)
$$

for all $u \in \mathbb{M}$. This shows that $\mathcal{A}(\mathbb{M})$ is uniformly bounded on $\mathbb{M}$.

On the other hand, let $t_{1}, t_{2} \in[0, T]$ be arbitrary with $t_{1}<t_{2}$. Then for any $u \in \mathbb{M}$, we get

$$
\begin{aligned}
& \left|(\mathcal{A} u)\left(t_{2}\right)-(\mathcal{A} u)\left(t_{1}\right)\right| \\
& =\mid\left(p\left(t_{2}\right)+\frac{1}{\Gamma(\beta)} \int_{0}^{t_{2}}\left(t_{2}-s\right)^{\beta-1} g(s, u(s)) d s\right) \\
& \times\left(\frac{1}{\Gamma(\alpha)} \int_{0}^{t_{2}}\left(t_{2}-s\right)^{\alpha-1} h(s, u(s)) d s+\theta\right) \\
& -\left(p\left(t_{1}\right)+\frac{1}{\Gamma(\beta)} \int_{0}^{t_{1}}\left(t_{1}-s\right)^{\beta-1} g(s, u(s)) d s\right) \\
& \times\left(\frac{1}{\Gamma(\alpha)} \int_{0}^{t_{1}}\left(t_{1}-s\right)^{\alpha-1} h(s, u(s)) d s+\theta\right) \mid \\
& \leq\left(\left|p\left(t_{2}\right)\right|+\frac{1}{\Gamma(\beta)} \int_{0}^{t_{2}}\left(t_{2}-s\right)^{\beta-1}|g(s, u(s))| d s\right) \\
& \times\left(\frac{1}{\Gamma(\alpha)}\left|\int_{0}^{t_{2}}\left(t_{2}-s\right)^{\alpha-1} h(s, u(s)) d s-\int_{0}^{t_{1}}\left(t_{1}-s\right)^{\alpha-1} h(s, u(s)) d s\right|\right) \\
& +\left(\left|p\left(t_{2}\right)-p\left(t_{1}\right)\right|+\frac{1}{\Gamma(\beta)} \mid \int_{0}^{t_{2}}\left(t_{2}-s\right)^{\beta-1} g(s, u(s)) d s\right. \\
& \left.-\int_{0}^{t_{1}}\left(t_{1}-s\right)^{\beta-1} g(s, u(s)) d s \mid\right)\left(\frac{1}{\Gamma(\alpha)} \int_{0}^{t_{1}}\left(t_{1}-s\right)^{\alpha-1}|h(s, u(s))| d s+|\theta|\right) .
\end{aligned}
$$

Thus,

$$
\begin{aligned}
& \left|(\mathcal{A} u)\left(t_{2}\right)-(\mathcal{A} u)\left(t_{1}\right)\right| \\
& \leq\left(\left|p\left(t_{2}\right)\right|+\frac{1}{\Gamma(\beta)} \int_{0}^{t_{2}}\left(t_{2}-s\right)^{\beta-1} G(s) d s\right) \frac{T^{\alpha}}{\Gamma(\alpha+1)}\left|\int_{t_{1}}^{t_{2}} H(s) d s\right| \\
& +\left(K_{p}\left|t_{2}-t_{1}\right|+\frac{T^{\beta}}{\Gamma(\beta+1)}\left|\int_{t_{1}}^{t_{2}} G(s) d s\right|\right) \\
& \times\left(\frac{1}{\Gamma(\alpha)} \int_{0}^{t_{1}}\left(t_{1}-s\right)^{\alpha-1} H(s) d s+|\theta|\right) \\
& \leq\left(\left|p\left(t_{2}\right)\right|+\frac{T^{\beta}\|G\|_{L^{1}}}{\Gamma(\beta+1)}\right) \frac{T^{\alpha}}{\Gamma(\alpha+1)}\left|\int_{t_{1}}^{t_{2}} H(s) d s\right| \\
& +\left(K_{p}\left|t_{2}-t_{1}\right|+\frac{T^{\beta}}{\Gamma(\beta+1)}\left|\int_{t_{1}}^{t_{2}} G(s) d s\right|\right)\left(\frac{T^{\alpha}\|H\|_{L^{1}}}{\Gamma(\alpha+1)}+|\theta|\right) \\
& =\left(\left|p\left(t_{2}\right)\right|+\frac{T^{\beta}\|G\|_{L^{1}}}{\Gamma(\beta+1)} \frac{T^{\alpha}}{\Gamma(\alpha+1)}\left|\rho\left(t_{2}\right)-\rho\left(t_{1}\right)\right|\right. \\
& +\left(\frac{T^{\alpha}\|H\|_{L^{1}}}{\Gamma(\alpha+1)}+|\theta|\right)\left(K_{p}\left|t_{2}-t_{1}\right|+\frac{T^{\beta}}{\Gamma(\beta+1)}\left|\sigma\left(t_{2}\right)-\sigma\left(t_{1}\right)\right|\right),
\end{aligned}
$$

where $\rho(t)=\int_{0}^{t} G(s) d s$ and $\sigma(t)=\int_{0}^{t} H(s) d s$. Since the functions $\rho$ and $\sigma$ are continuous on compact $[0, T]$, they are uniformly continuous. Hence, for $\varepsilon>0$, there exists a $\delta>0$ such that

$$
\left|t_{2}-t_{1}\right|<\delta \Longrightarrow\left|(\mathcal{A} u)\left(t_{2}\right)-(\mathcal{A} u)\left(t_{1}\right)\right|<\varepsilon
$$


for all $t_{1}, t_{2} \in[0, T]$ and $u \in \mathbb{M}$. This shows that $\mathcal{A}(\mathbb{M})$ is an equicontinuous set in $\mathbb{B}$. Now the set $\mathcal{A}(\mathbb{M})$ is uniformly bounded and equicontinuous set in $\mathbb{B}$, so it is a relatively compact by Arzela-Ascoli theorem. Thus, $\mathcal{A}$ is a compact operator on $\mathbb{M}$.

Step 3. We prove $\mathcal{A}$ is a continuous operator on $\mathbb{M}$ into $\mathbb{B}$. Let $\left\{u_{n}\right\}$ be a sequence in $\mathbb{M}$ converging to a point $u \in \mathbb{M}$. Then by the Lebesgue dominated convergence theorem, we obtain

$$
\begin{aligned}
\lim _{n \rightarrow \infty}\left(\mathcal{A} u_{n}\right)(t) & =\lim _{n \rightarrow \infty}\left[\left(p(t)+\frac{1}{\Gamma(\beta)} \int_{0}^{t}(t-s)^{\beta-1} g\left(s, u_{n}(s)\right) d s\right)\right. \\
& \left.\times\left(\frac{1}{\Gamma(\alpha)} \int_{0}^{t}(t-s)^{\alpha-1} h\left(s, u_{n}(s)\right) d s+\theta\right)\right] \\
& =\left(p(t)+\frac{1}{\Gamma(\beta)} \int_{0}^{t}(t-s)^{\beta-1} \lim _{n \rightarrow \infty} g\left(s, u_{n}(s)\right) d s\right) \\
& \times\left(\frac{1}{\Gamma(\alpha)} \int_{0}^{t}(t-s)^{\alpha-1} \lim _{n \rightarrow \infty} h\left(s, u_{n}(s)\right) d s+\theta\right) \\
& =\left(p(t)+\frac{1}{\Gamma(\beta)} \int_{0}^{t}(t-s)^{\beta-1} g(s, u(s)) d s\right) \\
& \times\left(\frac{1}{\Gamma(\alpha)} \int_{0}^{t}(t-s)^{\alpha-1} h(s, u(s)) d s+\theta\right) \\
& =(\mathcal{A} u)(t)
\end{aligned}
$$

for all $t \in[0, T]$. This shows that $\left\{\mathcal{A} u_{n}\right\}$ converges to $\mathcal{A} u$ pointwise on $[0, T]$. Moreover, the sequence $\left\{\mathcal{A} u_{n}\right\}$ is equicontinuous by a similar proof of Step 2. Therefore $\left\{\mathcal{A} u_{n}\right\}$ converges uniformly to $\mathcal{A} u$ and hence $\mathcal{A}$ is a continuous operator on $\mathbb{M}$.

Step 4. We show $\mathcal{A} u+\mathcal{B} v \in \mathbb{M}$ for all $u, v \in \mathbb{M}$.

For any $u, v \in \mathbb{M}$ and $t \in[0, T]$, we have

$$
\begin{aligned}
& |(\mathcal{A} u)(t)+(\mathcal{B} v)(t)| \\
& \leq \mid\left(p(t)+\frac{1}{\Gamma(\beta)} \int_{0}^{t}(t-s)^{\beta-1} g(s, u(s)) d s\right) \\
& \times\left(\frac{1}{\Gamma(\alpha)} \int_{0}^{t}(t-s)^{\alpha-1} h(s, u(s)) d s+\theta\right)+f(t, v(t)) \mid \\
& \leq\left(|p(t)|+\frac{1}{\Gamma(\beta)} \int_{0}^{t}(t-s)^{\beta-1}|g(s, u(s))| d s\right) \\
& \times\left(\frac{1}{\Gamma(\alpha)} \int_{0}^{t}(t-s)^{\alpha-1}|h(s, u(s))| d s+|\theta|\right)+|f(t, v(t))| \\
& \leq\left(K_{p} t+|p(0)|+\frac{1}{\Gamma(\beta)} \int_{0}^{t}(t-s)^{\beta-1} G(s) d s\right) \\
& \times\left(\frac{1}{\Gamma(\alpha)} \int_{0}^{t}(t-s)^{\alpha-1} H(s) d s+|\theta|\right)+|f(t, v(t))-f(t, 0)|+|f(t, 0)| \\
& \leq\left(K_{p} T+|p(0)|+\frac{T^{\beta}\|G\|_{L^{1}}}{\Gamma(\beta+1)}\right)\left(\frac{T^{\alpha}\|H\|_{L^{1}}}{\Gamma(\alpha+1)}+|\theta|\right)+K_{f}\|v\|+F_{0} \\
& \leq N .
\end{aligned}
$$

This shows that $\mathcal{A} u+\mathcal{B} v \in \mathbb{M}$ for all $u, v \in \mathbb{M}$.

Thus, all the conditions of Theorem 2.6 are satisfied and hence the operator equation $\mathcal{A} z+\mathcal{B} z=z$ has a solution in $\mathbb{M}$. Therefore, the initial value problem (1) has a mild solution defined on $[0, T]$. 
Theorem 3.4. Assume that $\left(A_{1}\right)-\left(A_{4}\right)$ are satisfied and

$$
\begin{aligned}
& {\left[\left(K_{p} T+|p(0)|+\frac{T^{\beta}\|G\|_{L^{1}}}{\Gamma(\beta+1)}\right) \frac{T^{\alpha} K_{h}}{\Gamma(\alpha+1)}\right.} \\
& \left.+\left(\frac{T^{\alpha}\|H\|_{L^{1}}}{\Gamma(\alpha+1)}+|\theta|\right) \frac{T^{\beta} K_{g}}{\Gamma(\beta+1)}+K_{f}\right]=\lambda<1 .
\end{aligned}
$$

Then the initial value problem (1) has a unique mild solution defined on $[0, T]$.

Proof. From Theorem 3.3 , it follows that the initial value problem (1) has a mild solution in $\mathbb{M}$. Hence, we need only to prove that the operator $\mathcal{A}+\mathcal{B}$ is a contraction on $\mathbb{M}$. In fact, for any $u, v \in \mathbb{M}$, we have

$$
\begin{aligned}
& |((\mathcal{A}+\mathcal{B}) u)(t)-((\mathcal{A}+\mathcal{B}) v)(t)| \\
& \leq\left(|p(t)|+\frac{1}{\Gamma(\beta)} \int_{0}^{t}(t-s)^{\beta-1}|g(s, u(s))| d s\right) \\
& \times\left(\frac{1}{\Gamma(\alpha)} \int_{0}^{t}(t-s)^{\alpha-1}|h(s, u(s))-h(s, v(s))| d s\right) \\
& +\left(\frac{1}{\Gamma(\beta)} \int_{0}^{t}(t-s)^{\beta-1}|g(s, u(s))-g(s, v(s))| d s\right) \\
& \times\left(\frac{1}{\Gamma(\alpha)} \int_{0}^{t}(t-s)^{\alpha-1}|h(s, v(s))| d s+|\theta|\right) \\
& +|f(t, u(t))-f(t, v(t))| \\
& \leq\left[\left(K_{p} T+|p(0)|+\frac{T^{\beta}\|G\|_{L^{1}}}{\Gamma(\beta+1)}\right) \frac{T^{\alpha} K_{h}}{\Gamma(\alpha+1)}\right. \\
& \left.+\left(\frac{T^{\alpha}\|H\|_{L^{1}}}{\Gamma(\alpha+1)}+|\theta|\right) \frac{T^{\beta} K_{g}}{\Gamma(\beta+1)}+K_{f}\right]\|u-v\| .
\end{aligned}
$$

Thus,

$$
\|(\mathcal{A}+\mathcal{B}) u-(\mathcal{A}+\mathcal{B}) v\| \leq \lambda\|u-v\| .
$$

Hence, the operator $\mathcal{A}+\mathcal{B}$ is a contraction mapping by (8). Therefore, by Banach's fixed point theorem, the initial value problem (1) has a unique mild solution in $\mathbb{M}$.

Example 3.5. Let us consider the following initial value problem

$$
\left\{\begin{array}{l}
{ }^{C} D_{0^{+}}^{\frac{1}{2}}\left(\frac{u(t)-\frac{1}{8} \sin u(t)}{\pi+\sin t+\frac{1 / 9}{\Gamma(1 / 3)} \int_{0}^{t}(t-s)^{-2 / 3} \sin u(s) d s}\right)=\frac{1}{7} \cos u(t), t \in[0,1], \\
u(0)=\frac{1}{8} \sin u(0)+\pi,
\end{array}\right.
$$

where $\alpha=\frac{1}{2}, \beta=\frac{1}{3}, T=1, \theta=1, f(t, u(t))=\frac{1}{8} \sin u(t), p(t)=\pi+\sin t, g(t, u(t))=\frac{1}{9} \sin u(t)$, $h(t, u(t))=\frac{1}{7} \cos u(t)$. Let $K_{f}=\frac{1}{8}, K_{p}=1, G(t)=\frac{1}{9}, H(t)=\frac{1}{7}$. Then hypotheses $\left(A_{1}\right)-\left(A_{3}\right)$ hold. Since

$$
K_{f}=\frac{1}{8}<1
$$

hence (5) holds. Therefore, by Theorem 3.3. the initial value problem (9) has a mild solution. Also, we have

$$
K_{g}=\frac{1}{9}, K_{h}=\frac{1}{7} \text { and } \lambda \simeq 0.957<1,
$$

then $\left(A_{4}\right)$ and (8) hold. So, by Theorem 3.4 (9) has a unique mild solution. 


\section{Higher order Caputo fractional integro-differential equations}

The method in Section 3 can be extended to the following initial value problem of nonlinear hybrid higher order Caputo fractional integro-differential equations

$$
\left\{\begin{array}{c}
{ }^{C} D_{0^{+}}^{\alpha}\left(\frac{u(t)-f(t, u(t))}{p(t)+\frac{1}{\Gamma(\beta)} \int_{0}^{t}(t-s)^{\beta-1} g(s, u(s)) d s}\right)=h(t, u(t)), t \in[0, T], \\
\left.\left(\frac{u(t)-f(t, u(t))}{p(t)+\frac{1}{\Gamma(\beta)} \int_{0}^{t}(t-s)^{\beta-1} g(s, u(s)) d s}\right)^{(k)}\right|_{t=0}=\theta_{k}, k=0, \ldots, n-1,
\end{array}\right.
$$

where $\alpha \in(n-1, n), \beta \in(n-1, n), \theta_{k} \in \mathbb{R}, p:[0, T] \rightarrow \mathbb{R}$ and $f, g, h:[0, T] \times \mathbb{R} \rightarrow \mathbb{R}$ are continuous functions with $p(t)+I_{0^{+}}^{\beta} g(t, u(t)) \neq 0$.

Lemma 4.1. $u \in C([0, T], \mathbb{R})$ is a mild solution of $[10)$ if $u$ satisfies

$$
\begin{aligned}
u(t) & =\left(p(t)+\frac{1}{\Gamma(\beta)} \int_{0}^{t}(t-s)^{\beta-1} g(s, u(s)) d s\right) \\
& \times\left(\frac{1}{\Gamma(\alpha)} \int_{0}^{t}(t-s)^{\alpha-1} h(s, u(s)) d s+\sum_{k=0}^{n-1} \frac{\theta_{k}}{k !} t^{k}\right)+f(t, u(t)) .
\end{aligned}
$$

The proof is similar to that of Lemma 3.2 and hence, we omit it.

Theorem 4.2. Suppose that hypotheses $\left(A_{1}\right)-\left(A_{3}\right)$ and (5) hold. Then (10) has a mild solution.

The proof is similar to that of Theorem 3.3 and hence, we omit it.

Theorem 4.3. Suppose that $\left(A_{1}\right)-\left(A_{4}\right)$ are satisfied and

$$
\begin{aligned}
& {\left[\left(K_{p} T+|p(0)|+\frac{T^{\beta}\|G\|_{L^{1}}}{\Gamma(\beta+1)}\right) \frac{T^{\alpha} K_{h}}{\Gamma(\alpha+1)}\right.} \\
& \left.+\left(\frac{T^{\alpha}\|H\|_{L^{1}}}{\Gamma(\alpha+1)}+\sum_{k=0}^{n-1} \frac{\left|\theta_{k}\right|}{k !} T^{k}\right) \frac{T^{\beta} K_{g}}{\Gamma(\beta+1)}+K_{f}\right]=\Lambda<1 .
\end{aligned}
$$

Then (10) has a unique mild solution.

The proof is similar to that of Theorem 3.4 and hence, we omit it.

\section{Conclusion}

In the current paper, we have studied the existence and uniqueness of mild solutions for initial value problems of nonlinear hybrid Caputo fractional integro-differential equations. We have presented the existence and uniqueness theorems for the initial value problems (1) and (10) under some sufficient conditions due to the Krasnoselskii and Banach fixed point theorems. The main results have been well illustrated with the help of an example. Our results in this paper have been extended and improved some wellknown results. Acknowledgements. The authors are grateful to the referees for their valuable comments which have led to improvement of the presentation. 


\section{References}

[1] A. Ardjouni, A. Djoudi, Approximating solutions of nonlinear hybrid Caputo fractional integro-differential equations via Dhage iteration principle, Ural Mathematical Journal 5(1) (2019) 3-12.

[2] A. Ardjouni, A. Djoudi, Initial-value problems for nonlinear hybrid implicit Caputo fractional differential equations, Malaya Journal of Matematik 7 (2019) 314-317.

[3] A. Ardjouni, A Djoudi, Existence and uniqueness of solutions for nonlinear hybrid implicit Caputo-Hadamard fractional differential equations, Results in Nonlinear Analysis 2(3) (2019) 136-142.

[4] M. Benchohra, S. Hamani, S.K. Ntouyas, Boundary value problems for differential equations with fractional order, Surveys in Mathematics its Applications 3 (2008) 1-12.

[5] B. Bordj, A. Ardjouni, Periodic and asymptotically periodic solutions in nonlinear coupled Volterra integro-dynamic systems with in nite delay on time scales, Advances in the Theory of Nonlinear Analysis and its Applications 5(2) (2021) 180-192.

[6] B.C. Dhage, Hybrid fixed point theory in partially ordered normed linear spaces and applications to fractional integral equations, Differ. Equ. Appl. 5 (2013) 155-184.

[7] B.C. Dhage, Basic results in the theory of hybrid differential equations with mixed perturbations of second type, Funct. Differ. Equ. 19 (2012) 87-106.

[8] B.C. Dhage, A fixed point theorem in Banach algebras with applications to functional integral equations, Kyungpook Math. J. 44 (2004) 145-155.

[9] B.C. Dhage, S.B. Dhage, S.K. Ntouyas, Approximating solutions of nonlinear second order ordinary differential equations via Dhage iteration principle. Malaya J. Mat. 4(1) (2016) 8-18.

[10] B.C. Dhage, G.T. Khurpe, A.Y. Shete, J.N. Salunke, Existence and approximate solutions for nonlinear hybrid fractional integro-differential equations, International Journal of Analysis and Applications 11(2) (2016) 157-167.

[11] B.C. Dhage, V. Lakshmikantham, Basic results on hybrid differential equations, Nonlinear Anal. Hybrid Syst. 4 (2010) 414-424.

[12] M. Haoues, A. Ardjouni, A. Djoudi, Existence, uniqueness and monotonicity of positive solutions for hybrid fractional integro-differential equations, Asia Mathematika 4(3) (2020) 1-13.

[13] A.A. Kilbas, H.M. Srivastava, J.J. Trujillo, Theory and Applications of Fractional Differential Equations, Elsevier Science B. V., Amsterdam, (2006).

[14] H. Lu, S. Sun, D. Yang, Theory of fractional hybrid differential equations with linear perturbations of second type, Bound. Value Probl. 2013(23) (2013).

[15] D.R. Smart, Fixed Point Theorems, Cambridge Tracts in Mathematics, Cambridge University Press, London-New York, (1974).

[16] S. Sun, Y. Zhao, Z. Han, The existence of solutions for boundary value problem of fractional hybrid differential equations, Commun. Nonlinear Sci. Numer. Simul. 17 (2012) 4961-4967.

[17] Y. Zhao, S. Sun, Z. Han, Theory of fractional hybrid differential equations, Comput. Math. Appl. 62 (2011) $1312-1324$.

[18] Y. Zhao, S. Sun, Z. Han, Positive solutions for boundary value problems of nonlinear fractional differential equations, Appl. Math. Comput. 217 (2011) 6950-6958.

[19] Y. Zhao, Y. Sun, Z. Liu, Basic theory of differential equations with mixed perturbations of the second type on time scales, Adv. Differ. Equa. 2019(268) (2019).

[20] Y. Zhao, Y. Sun, Z. Liu, Y. Wang, Solvability for boundary value problems of nonlinear fractional differential equations with mixed perturbations of the second type, AIMS Mathematics 5(1) (2019) 557-567. 